\title{
Localización de empresas usando lógica difusa: estrategia para su posicionamiento
}

\author{
Location of companies using fuzzy logic: Strategy for positioning \\ Martha Beatriz Flores Romero ${ }^{1}$, Cuauhtémoc Guerrero Dávalos ${ }^{1}$, \\ Federico González Santoyo ${ }^{2}$ \\ ${ }^{1}$ Instituto Iberoamericano de Desarrollo Empresarial (INIDEM), México \\ ${ }^{2}$ Universidad Michoacana de San Nicolás de Hidalgo, México
}

Recibido el 15 de febrero de 2018; aceptado el 7 de enero de 2019

Disponible en Internet el: 11 de enero de 2019

\section{Resumen}

Esta investigación, consiste en determinar la ubicación óptima en la certeza e incertidumbre de una empresa dedicada a la producción de derivados del aguacate en el Estado de Michoacán, para ello toma en consideración factores objetivos y subjetivos. En este estudio se usará como base la metodología propuesta por Brown y Gibsosn (1972)- (B y G), la misma es presentada en teoría clásica, además se hace el análisis en un ambiente de incertidumbre usando lógica difusa, este enfoque no se ha reportado en la literatura especializada para el tratamiento de la metodología de referencia. Para la obtención de información (datos) se incorpora a la metodología de (B y G) apoyada por un panel de 5 expertos. Los resultados obtenidos usando lógica difusa, son eficientes y eficaces, se obtiene la ubicación óptima en la comunidad de San Juan Nuevo Parangaricutiro. De lo anterior se tiene que la aplicación de (ByG) usando números borrosos triangulares (NBT) en el estudio de localización de empresas, en el estudio se obtiene información no proporcionada de origen que es muy valiosa para fortalecer la toma de decisiones y diseño e implantación de planes estratégicos de desarrollo empresarial.

\section{Código JEL: C69, C83, L1}

Palabras clave: Localización; Óptimo; Factor básico localizacional; Empresa; Competitividad;

Lógica difusa

\footnotetext{
*Autor para correspondencia. Correo electrónico: cmgj6819@hotmail.com (C. Guerrero Dávalos).

La revisión por pares es responsabilidad de la Universidad Nacional Autónoma de México. 


\begin{abstract}
This research consists of determining the optimal location in the certainty and uncertainty (diffuse environment) of a company dedicated to the production of avocado derivatives in the State of Michoacán taking into account objective and subjective factors. For this, the methodology proposed by Brown and Gibsosn (1972) - (B and G) will be used, it is presented in classical theory, and the analysis is done in an uncertainty environment using fuzzy logic, this approach has not been reported in the specialized literature for the treatment of the reference methodology. To obtain information (data) a panel of 5 experts is incorporated into the methodology of (B and $\mathrm{G}$ ). The results obtained using fuzzy logic are efficient and effective, obtaining the optimum location in the community of San Juan Nuevo Parangaricutiro. From the above it is necessary that the application of (ByG) using NBT in the study of location of companies, provides information not provided of origin that is very valuable to strengthen the decision making and design and implementation of strategic business development plans.
\end{abstract}

JEL code: $\mathrm{C} 69, \mathrm{C} 83, \mathrm{~L} 1$

Keywords: Localization; Optimal; Basic localizational factor; Company; Competitiveness; Fuzzy logic

\title{
Introducción
}

En las empresas tanto públicas como privadas, productoras de bienes de consumo final, intermedio y de capital y/o servicios, la determinación del sitio (localidad) en que deben ser instaladas (localizadas) es un elemento estratégico para su desarrollo económico de cualquier país, región o localidad. De acuerdo con González Santoyo et al (1985), Flores Garrido L. et al. (2016). La localización de una empresa (planta) es representada por el sitio o (unidad de área geográfica, Ai, i = 1,2,..,n) en el que desarrollará sus actividades de producción u ofrecimiento de servicios, así como sus transacciones comerciales, de tal forma que se garantice el mayor nivel de rentabilidad financiera y una aceptación social plena, de acuerdo con la ONU (1958), Bojic S. et al. (2018), la localización más adecuada para una empresa está orientada a la selección de una localidad que garantice el mayor nivel de beneficio económico (rentabilidad), desde la perspectiva de inversión privada y de aquella localidad que permita tener el menor nivel de costo de operación si se considera un enfoque social.

Para la definición del área geográfica (nivel macro-micro) (Ai), es importante considerar, si su localización está orientada hacia el mercado (ubicación cercana en la que se encuentra el área de mercado), o bien si está orientada a las materias primas e insumos (ubicación cercana a estos), se tratará de insumos y materias primas de fuerte incidencia económica.

Uno de los elementos relevantes a considerar en este análisis, son las políticas de descentralización marcadas por el Estado, se tendrán casos en los que se quiera desarrollar alguna área del país, estado o municipio, creando al mismo tiempo, incentivos fiscales o de otro tipo que son benéfico para el inversionista. 
Esto es relevante ya que las organizaciones actuales presentan grandes cambios en el entorno. Para Castro García et al. (2010), el mercado es muy competitivo, y la globalización hoy día es una realidad. Esto implica para cualquier empresario operar con las mismas condiciones de competencia. Un factor fundamental en el desarrollo empresarial lo representa la localización eficiente y eficaz de las empresas, González Santoyo F. et al. (2011), Jeong J.S., Ramírez G.A. (2018), consideran que deberá incorporarse un plan estratégico de desarrollo multifactorial para atender la localización, así como las condiciones de las posibles áreas de interés dadas por (Ai), así como las del entorno.

De acuerdo con Machuca et al. (1994). Generalmente las decisiones de localización de una planta (empresa) se toman una sola vez en la historia de las empresas, sin descartar la posibilidad de la reolocalización por no cumplir adecuadamente con las necesidades que requiere la planta para su adecuada operación, lo que implicaría una baja en su rentabilidad operativa.

De acuerdo con Tawfik et al. (1993), entre los problemas más comunes que se tienen cuando no se localiza adecuadamente una empresa son: alejamiento con los mercados de consumo, dificultades de los abastecimientos de materias primas e insumos y servicios, problemas de disponibilidad de mano de obra calificada, incremento en los costos de transporte.

Así mismo Garret (1973) y Dilowrth (1993), establecen que las causas más importantes que se tienen al localizar una empresa son: cambios en los niveles de la demanda, cambios significativos en la distribución de la demanda, altos niveles en los costos de transporte de materiales e insumos, necesidad de cambio de lugar por rechazo de la comunidad o por cuestiones de tipo ambiental, cambio de sitio por problemas de seguridad, agotamiento de sus fuentes de abastecimiento.

En el proceso de solución del problema de localización de empresas se tienen tres componentes a definir, las cuales son: la selección de la región, la determinación de la localidad dentro de la región y la selección del sitio específico en el que se ubicará la empresa. Esto estará asociado a lo que se le llama Macrolocalización y Microlocalización.

De acuerdo con Medina J.R. et al (2009), la Macrolocalización, tiene como objetivo la determinación de la región o territorio en el que se tiene interés la ubicación de la planta, el mismo puede tener un orden internacional, nacional, regional o local por lo que es de fundamental importancia la determinación de los factores básicos localizacionales \{FBLi; $\mathrm{i}=1,2, \ldots, \mathrm{n}\}$ de acuerdo con su alcance geográfico, para Kavita D., Shiv Prasad Y (2013) entre los más importantes se pueden considerar:

Aspectos socioeconómicos y culturales: población total; población económicamente activa; sectores de la economía (ramas de actividad); sueldos y salarios; educación; salud pública; estructura de poder vigente.

Infraestructura: vías de comunicación; electrificación; obras de irrigación; redes de agua potable; centros de acopio y almacenamiento; tecnologías de comunicación. 
Aspectos Institucionales: régimen de propiedad; instituciones crediticias; programas de desarrollo gubernamental.

Análisis de mercados de consumo: dispersión geográfica de los demandantes.

Disponibilidad y costo de: materias primas; insumos auxiliares; mano de obra: asistencia técnica; energía eléctrica: agua; combustibles.

Costos de transporte de: materias primas e insumos auxiliares; productos y subproductos.

Factores geográficos: fenómenos ecológicos; condiciones topográficas.

Factores Institucionales: políticas de descentralización; políticas de planeación por sector de la economía; incentivos fiscales.

Para González Santoyo F. (2005). La Microlocalización, tiene como objetivo realizar el estudio que permita definir la localidad en que se tenga el lugar exacto en el que se deba ubicar la planta (empresa), buscando que este sitio sea el que garantice la más alta rentabilidad financiera o el mayor nivel de beneficio- costo $(\mathrm{B} / \mathrm{C})$. El sitio seleccionado será aquel en el que se cumpla de forma más eficiente y eficaz el conjunto de factores básicos localizacionales expresados como $\{\mathrm{FBLi} ; \mathrm{i}=1,2, \ldots, \mathrm{n}\}$.

La mayoría de modelos existentes en la literatura usados para la localización de empresas incluyen solamente factores de tipo cuantitativo. Los pocos que incluyen factores de tipo cualitativo usan escalas ordinales para su medición las cuales son totalmente arbitrarias. Esto implica que este tipo de metodologías son rígidas y representan muy poca flexibilidad para representar la realidad, por ello es de importancia hacer uso de nuevas metodologías como la Lógica Difusa.

Los modelos matemáticos usados actualmente para tomar decisiones eficientes y eficaces de localización de una empresa son basados en la teoría clásica de conjuntos y de Investigación de Operaciones que, bajo una lógica bivalente, consideran que un elemento solo tiene las opciones de estar o no dentro del mismo. De acuerdo con Klir et al. (1995), S. Melkote, M.Daskin (2001), A.Klose. A.Drexl (2005), se tiene que el paso de los elementos de un conjunto a otro se realiza de forma gradual y no dando pasos sin orden y acelerados. Por ejemplo a una localidad a la que en un inicio se le consideraba no adecuada para la ubicación de una empresa, si a dicha localidad se le van haciendo mejoras graduales, cambios en la estructura demográfica, cambios en su entorno socioeconómico, entre otros, puede ir transformándose hasta convertirse en la mejor alternativa posible para localizar una empresa, esto desde la perspectiva de la apreciación del ser humano al percibir que está mejorando dicha localidad.

En otro sentido al tomar decisiones en función de cómo piensa el ser humano, pareciera no trabajar de forma directa con números asociados a variables medibles. De la actuación del pensamiento humano surge la teoría de la Lógica Difusa, la cual reconoce y permite expresar, las transiciones graduales entre la membresía y no membresía de los elementos integrados en los conjuntos. Esto es formalizado haciendo uso de la función de membresía, lo que permite 
tener la medida del grado de compatibilidad existente entre un valor observado y el concepto al cual se asocia, este enfoque permitirá tener una mejor aproximación a la realidad, en este trabajo se incorporan factores localizacionales subjetivos.

En la teoría de localización de empresas para Fazel Zarandi M, Beck J.C. (2012), Antony B., et al. (2010), existen una infinidad de métodos. En el presente trabajo se estudiará el proceso de localización de empresas tomando como referencia el Estado de Michoacán México, para ello, se usara como metodología de análisis la propuesta por Brown y Gibson (ByG) de 1972. El objetivo es realizar la propuesta de un método de apoyo a la toma de decisiones en el área de localización de empresas en la que se incorpore un conjunto de factores básicos localizacionales expresados por $\{\mathrm{FBLi} ; \mathrm{i}=1,2, \ldots, \mathrm{n}\}$ clásicos, en los cuales para su cualificación se incorpora un panel de 5 expertos los cuales hacen uso del método Delphi para la obtención de la información que se usará en el análisis, estos definen el nivel de importancia de cada factor. En el proceso de la asignación de su nivel de peso (importancia-ponderación). En la investigación a $(B y G)$ se le hace una extensión a lo existente hoy día en la literatura especializada haciéndolo apto para que evalué la localización de empresas en un ambiente de incertidumbre (difuso).

La presente investigación se encuentra organizada de acuerdo a: 1. Introducción, se presenta un panorama del tema de localización de plantas, 2. Metodología propuesta, se presenta la metodología de interés en un ambiente determinista, 3. Análisis de caso, se evalúa en un ambiente determinista, de igual forma se hace la extensión de la metodología para ser usada en un ambiente incierto (difuso), Conclusiones y Bibliografía.

\section{Metodología Propuesta}

Para el análisis de la localización de una empresa en este trabajo se propone el método de (ByG), en el método se permite hacer la combinación de factores objetivos posibles de cuantificar con factores subjetivos que se pueden valorar en términos relativos. Para que este método sea adecuado se consideran las localidades de interés tomando como referencia los niveles de país, región, ciudades, municipios, etc, que cumplan con el conjunto de factores básicos localizacionales $\{\mathrm{FBLi}, \mathrm{i}=1,2, \ldots, \mathrm{n}\}$ que se requieren para la localización de la empresa que es de interés, descartando todas aquellas propuestas que no cumplen en un nivel mínimo establecido por el panel de expertos, dichos factores por su complejidad para estimar su importancia son clasificados en factores objetivos y subjetivos. La metodología de referencia es integrada en las siguientes etapas:

Realizar la asignación de un valor relativo a cada factor objetivo (FOi, i = 1,2,...n) para cada localidad viable y de interés usada en el análisis.

Realizar la estimación de un valor relativo de cada factor subjetivo (FSi, i = 1,2,...n) para cada localidad de interés incorporada en el análisis. 
Combinar los factores objetivos y subjetivos, asignándoles una ponderación relativa, para obtener una medida de preferencia de la localización $\left(\mathrm{MPL}_{\mathrm{i}}, \mathrm{i}=1,2, \ldots . \mathrm{n}\right)$.

Seleccionar la ubicación que en la evaluación tenga la máxima medida de preferencia de localización obtenida en el punto (3).

Para operacionalizar la metodología en la etapa (1). El valor relativo de los factores objetivos $\left(\mathrm{FO}_{\mathrm{i}}, \mathrm{i}=1,2, \ldots \mathrm{n}\right)$. Se hace posible cuantificándolos en términos de costo, esto permitirá obtener el costo total anual de cada alternativa de localización (Ci). Por lo que (FOi, $\mathrm{i}=1,2, \ldots . \mathrm{n})$ se determina multiplicando $\left(\mathrm{C}_{\mathrm{i}}\right)$ de cada localidad de interés considerada en el estudio por la suma de los costos recíprocos $(1 / \mathrm{Ci})$ y tomando el recíproco de su resultado, dicho cálculo de hace usando la ecuación (1) como se muestra:

$$
F O_{i}=\left[C_{i} \sum_{i=1}^{n}\left(\frac{1}{C_{i}}\right)\right]^{-1}
$$

Dónde:

$\mathrm{C}_{\mathrm{i}}=$ costo de cada factor básico localizacional.

La suma de los $\left(\mathrm{FO}_{\mathrm{i}}, \mathrm{i}=1,2, \ldots \mathrm{n}\right)$ siempre será como máximo igual a la unidad, por lo que se asume que cada uno de ellos es siempre un término relativo entre las distintas alternativas usadas en el análisis de localización.

En la etapa (2). El valor relativo de los factores subjetivos $\left(\mathrm{FS}_{\mathrm{i}}, \mathrm{i}=1,2, \ldots \mathrm{n}\right)$, es obtenido haciendo uso de la ecuación (2) descrita como:

$$
F S_{i}=\sum_{j=1}^{n} R_{i j} W_{j}
$$

Dónde:

$\mathrm{W}_{\mathrm{j}}$ = índice de importancia relativa de cada factor localizacional respecto a los otros considerados.

$\mathrm{R}_{\mathrm{ij}}=$ puntaje relativo asignado por la evaluación de las localizaciones en comparaciones pareadas.

Para ello, se deben considerar las condiciones siguientes:

$$
\left(0 \leq R_{i j} \leq 1, \sum_{i=1}^{n} R_{i j}=1\right)
$$

En el análisis la realización de comparaciones pareadas el valor de 1, se asigna cuando el factor básico localizacional es más relevante y 0 , se asigna al menos importante, para el caso en que se tienen $\left\{\mathrm{FBL}_{\mathrm{i}}, \mathrm{i}=1,2, \ldots, \mathrm{n}\right\}$ que se consideran con importancia igual a ambos se les asigna el valor 1 . 
Para la realización de la etapa (3), en el cálculo de la medida de preferencia de localización (MPL), primeramente deberán haber sido valorados los factores objetivos y subjetivos de localización, hecho esto se calcula la medida de localización aplicando la ecuación (3).

$$
M P L_{I}=K\left(F O_{i}\right)+(1-K)\left(F S_{i}\right)
$$

La importancia relativa que existe entre los factores objetivos y subjetivos de la localización se obtiene asignándole una ponderación (K) a uno de los factores y su complemento (1-K) al otro tipo de factores, de tal forma que se expresa entre ellos una importancia relativa. Para el caso esto se hace tomando en consideración en una escala de cero a diez cuantas veces son más importantes un tipo de factores con respecto a los otros. Por lo que el mejor lugar para ser seleccionado para localizar la empresa será el que cumpla con el criterio del Max.\{MPLi \}. Esto implica que un cambio de los niveles de ponderación asignados a los factores puede llevar a otra(s) decisión(es), porque implicaría hacer la evaluación usando diferentes escenarios.

Para el cálculo la etapa (4). Se hace uso de la ecuación (4), a través de su evaluación se logra la selección del sitio óptimo para localizar la planta.

$$
\max .\left\{M P L_{i}\right\}=K\left(F O_{i}\right)+(1-K)\left(F S_{i}\right)
$$

\section{Análisis de caso}

\section{Análisis usando teoría clásica (uso de números nítidos)}

Para el análisis se tomará el caso de la empresa denominada corporativo aguacatero de la Meseta Tarasca, dicha organización tiene interés en realizar inversiones en el Estado de Michoacán México. La actividad económica quiere iniciarla con una empresa dedicada a la producción de derivados del aguacate. El citado corporativo tiene interés en 4 sitios para realizar la localización de la empresa en análisis, para ello se tiene preferencia por las poblaciones de Uruapan (U), Apatzingan (A), Morelia (M), y San Juan Nuevo Parangaricutiro (SJN). El panel de expertos ha considera que en dichas localidades se tiene cumplimiento de la mayor parte de los factores básicos localizacionales $\left\{\mathrm{FBL}_{\mathrm{i}}, \mathrm{i}=1,2, \ldots, \mathrm{n}\right\}$, así como la aceptación de la población de cada localidad que es fundamental para conservar la estabilidad y bienestar social al instalar dicha empresa en cualquiera de ellas. Para la realización del análisis se hace uso de la metodología descrita en el apartado 2.

Para la etapa (1): Los costos (Ci) para cada localidad son expresados en por unidad (p.u.\$), se observa que los mismos son diferentes para cada localidad, haciendo un análisis de costeo para cada uno de los factores localizacionales de referencia se obtienen los mostrados en la tabla \# 1: 
Tabla 1

Costos Asociados

\begin{tabular}{lcccc}
\hline CONCEPTO $\backslash$ Costo $(\mathrm{p} . \mathrm{u}-\$)$ & $\mathrm{U}$ & $\mathrm{A}$ & $\mathrm{M}$ & $\mathrm{SJN}$ \\
Materias primas & 8.5 & 9.5 & 10.0 & 8.5 \\
Mano de obra & 9.4 & 10.0 & 8.5 & 9.0 \\
Transporte & 8.6 & 9.6 & 10.0 & 8.0 \\
Energía Eléctrica & 9.4 & 9.4 & 9.5 & 9.4 \\
Costo de terreno & 10.0 & 8.5 & 8.0 & 9.0 \\
Abastecimiento de $\mathrm{H}_{2} \mathrm{O}$ & 7.0 & 7.0 & 7.5 & 7.0 \\
Insumos & 8.5 & 9.0 & 7.0 & 8.5 \\
TOTAL $\left(\mathrm{C}_{\mathrm{i}}\right)$ & 61.4 & 63 & 60.5 & 59.4 \\
Reciproco $\left(1 / \mathrm{C}_{\mathrm{i}}\right)$ & 0.01628 & 0.01587 & 0.01652 & 0.01683 \\
\hline
\end{tabular}

Fuente: elaboración propia.

El total de $\left(1 / \mathrm{C}_{\mathrm{i}}\right)$ es igual a 0.0655 , esto será la base para el cálculo del factor de calificación objetiva para cada localidad, el cual es expresado en la tabla \# 2, como se muestra a continuación:

Tabla 2

Factor de Calificación Objetiva

\begin{tabular}{ccc}
\hline Localidad & $\mathrm{FO}_{\mathrm{i}}$ \\
$\mathrm{U}$ & $0.01628 / 0.0655$ & 0.248549618 \\
$\mathrm{~A}$ & $0.01587 / 0.0655$ & 0.242290076 \\
$\mathrm{M}$ & $0.01652 / 0.0655$ & 0.25221374 \\
SJN & $0.01683 / 0.0655$ & 0.256946565 \\
Total & & 1.0 \\
\hline
\end{tabular}

Fuente: elaboración propia.

Etapa (2): para el análisis de los factores subjetivos $\left(\mathrm{FS}_{\mathrm{i}}\right)$, se hace necesario calcular el índice de importancia relativa $\left(\mathrm{W}_{\mathrm{j}}\right)$, así como el índice de ordenamiento jerárquico $\left(\mathrm{R}_{\mathrm{ij}}\right)$. Para ello se hace uso de la ecuación (2):

En el estudio se consideran como factores subjetivos la educación, el clima, la vivienda, la aceptación de la población para la instalación de la empresa en la localidad. Para ello en las evaluaciones pareadas se asignará el valor de (1) al factor más relevante y (0) al menos importante, para el caso en que se tenga el mismo nivel de importancia a todos los factores se asignará (1).

El cálculo iniciará con la determinación del factor de importancia relativa $\left(\mathrm{W}_{\mathrm{j}}\right)$, los mismos 
son establecidos por el panel de expertos, y calculados usando el criterio de cálculo de probabilidad de un evento P(A), establecido por Laplace en el siglo XVII, los valores obtenidos para $\left(\mathrm{W}_{\mathrm{j}}\right)$ se muestran en la tabla \# 3 .

Tabla 3

Cálculo del factor de importancia relativa

\begin{tabular}{|c|c|c|c|c|c|c|}
\hline \multirow[t]{2}{*}{ Factor (i) } & \multicolumn{4}{|c|}{ Comparaciones Pareadas } & \multirow{2}{*}{$\begin{array}{c}\text { Suma de Prefe- } \\
\text { rencia }\end{array}$} & \multirow[t]{2}{*}{$\mathrm{W}_{\mathrm{j}}$} \\
\hline & $\mathrm{U}$ & A & M & SJN & & \\
\hline Educación & 1 & 0 & 0 & 1 & 2 & $2 / 7=0.2858$ \\
\hline Clima & 0 & 1 & 1 & 0 & 2 & $2 / 7=0.2858$ \\
\hline Vivienda & 0 & 0 & 0 & 1 & 1 & $1 / 7=0.14286$ \\
\hline Aceptación de la Comunidad & 1 & 0 & 0 & 1 & 2 & $2 / 7=0.2858$ \\
\hline TOTAL & & & & & 7 & 1.0 \\
\hline
\end{tabular}

Fuente: elaboración propia.

De igual forma usando el criterio establecido para la obtención de los resultados de la tabla anterior se calcula el Índice de Ordenamiento Jerárquico (Rij), que representa la importancia relativa de cada factor localizacional en función del sitio de interés. El análisis para cada factor se muestra a continuación.

Tabla 4

Para Educación

\begin{tabular}{|c|c|c|c|c|c|c|}
\hline \multirow[t]{2}{*}{ FACTOR (i) } & \multicolumn{4}{|c|}{ Comparaciones Pareadas } & \multirow{2}{*}{$\begin{array}{c}\text { Suma de Prefe- } \\
\text { rencia }\end{array}$} & \multirow[t]{2}{*}{$\mathrm{R}_{\mathrm{iU}}$} \\
\hline & 1 & 2 & 3 & 4 & & \\
\hline A & 1 & 0 & 0 & 0 & 1 & $1 / 6=0.16666$ \\
\hline $\mathrm{U}$ & 0 & 1 & 1 & 0 & 2 & $2 / 6=0.33333$ \\
\hline M & 0 & 0 & 0 & 0 & 0 & 0 \\
\hline SJN & 1 & 1 & 1 & 0 & 3 & 0.5 \\
\hline TOTAL & & & & & 6 & 1.0 \\
\hline
\end{tabular}

Fuente: elaboración propia. 
Tabla 5

Para Clima

\begin{tabular}{lcccccc}
\hline & Factor (i) & \multicolumn{3}{c}{ Comparaciones Pareadas } & \multicolumn{2}{c}{ Suma de Prefe- } \\
& 1 & 2 & 3 & 4 & $\mathrm{R}_{\mathrm{iE}}$ \\
$\mathrm{A}$ & 0 & 1 & 0 & 1 & 2 & 0.28571 \\
$\mathrm{U}$ & 0 & 1 & 0 & 0 & 1 & 0.1426 \\
$\mathrm{M}$ & 0 & 0 & 0 & 0 & 0 & 0 \\
SJN & 1 & 1 & 1 & 1 & 4 & 0.57142 \\
\hline TOTAL & & & & 7 & 1.0 \\
\hline
\end{tabular}

Fuente: elaboración propia.

Tabla 6

Para Vivienda

\begin{tabular}{|c|c|c|c|c|c|c|}
\hline \multirow[t]{2}{*}{ Factor (i) } & \multicolumn{4}{|c|}{ Comparaciones Pareadas } & \multirow{2}{*}{$\begin{array}{l}\text { Suma de Prefe- } \\
\text { rencia }\end{array}$} & \multirow[t]{2}{*}{$\mathrm{R}_{\mathrm{i}}$} \\
\hline & 1 & 2 & 3 & 4 & & \\
\hline A & 1 & 0 & 0 & 0 & 1 & 0.16666 \\
\hline $\mathrm{U}$ & 0 & 1 & 0 & 0 & 1 & 0.16666 \\
\hline M & 1 & 0 & 0 & 1 & 2 & 0.33333 \\
\hline SJN & 1 & 0 & 0 & 1 & 2 & 0.33333 \\
\hline TOTAL & & & & & 6 & 1.0 \\
\hline
\end{tabular}

Fuente: elaboración propia.

Tabla 6

Para Aceptación de la Comunidad

\begin{tabular}{lcccccc}
\hline & Factor (i) & \multicolumn{1}{c}{ Comparaciones Pareadas } & \multicolumn{2}{c}{ Suma de Prefe- } & \multicolumn{1}{c}{$\mathrm{R}_{\mathrm{iC}}$} \\
& 1 & 2 & 3 & 4 & & rencia \\
$\mathrm{A}$ & 0 & 0 & 0 & 1 & 1 & 0.1428571 \\
$\mathrm{U}$ & 1 & 1 & 0 & 0 & 2 & 0.285714 \\
$\mathrm{M}$ & 0 & 0 & 0 & 0 & 0 & 0 \\
SJN & 1 & 1 & 1 & 1 & 4 & 0.571428 \\
\hline TOTAL & & & & & 7 & 1.0 \\
\hline
\end{tabular}

Fuente: elaboración propia. 
Por lo que el cálculo de los diferentes indicadores de Índice de Ordenamiento Jerárquico $\left(\mathrm{R}_{\mathrm{ij}}\right)$ y del factor de importancia relativa $\left(\mathrm{W}_{\mathrm{j}}\right)$, quedan como se muestra en la tabla \# 7 :

Tabla 7

Índice de Ordenamiento Jerárquico

\begin{tabular}{|c|c|c|c|c|c|c|}
\hline \multirow[t]{2}{*}{ Factor (i) } & & \multicolumn{4}{|c|}{$\left(\mathrm{R}_{\mathrm{ij}}\right)$} & \multirow[t]{2}{*}{$\mathrm{W}_{\mathrm{j}}$} \\
\hline & & $\mathrm{U}$ & A & $\mathrm{M}$ & SJN & \\
\hline Educación & & 0.333333 & 0.166666 & 0 & 0.5 & $2 / 7=0.2858$ \\
\hline Clima & & 0.142666 & 0.285711 & 0 & 0.571422 & $2 / 7=0.2858$ \\
\hline Vivienda & & 0.166666 & 0.166666 & 0.33333 & 0.333333 & $1 / 7=0.14286$ \\
\hline $\begin{array}{l}\text { Aceptación de la } \\
\text { munidad }\end{array}$ & Co- & 0.285714 & 0.142857 & 0 & 0.571428 & $2 / 7=0.2858$ \\
\hline TOTAL & & & & & & 1.0 \\
\hline
\end{tabular}

Fuente: elaboración propia.

Para concluir la etapa (2), el cálculo de los Factores Subjetivos (FSi) para cada localidad se hace aplicando la siguiente ecuación (2) descrita en el apartado (2) de metodología.

Usando como ejemplo de esta aplicación se usará la localidad de (SJN) se tiene:

$\mathrm{FS}_{\mathrm{SJN}}=0.5(0.2858)+0.57142(0.2858)+0.3333(0.14286)+0.571428(0.2858)=0.517144$

1. Usando la misma metodología que en el ejemplo anterior. El resumen de los cálculos para las diferentes localidades establecidas como de interés para el estudio se obtienen los resultados mostrados en la tabla \#8 como se muestra:

Tabla 8

Factores Subjetivos (FSi)

\begin{tabular}{lc}
\hline (FSi) & Nivel De Importancia \\
SJN & 0.5171441 \\
$\mathrm{M}$ & 0.04762 \\
$\mathrm{U}$ & 0.2414872 \\
$\mathrm{~A}$ & 0.193927 \\
$\mathrm{\Sigma}$ & 1.0 \\
\hline
\end{tabular}

Fuente: elaboración propia.

Para la obtención de lo establecido en la etapa (3). La medida de preferencia de localización es calculada haciendo uso de la ecuación (3) descrita en el apartado (2). 
Para la asignación del nivel de ponderación (K) a los factores objetivos (FOi) y (1-K) a los factores subjetivos (FSi). En el análisis, el panel de expertos considera y recomienda que los factores objetivos son 2.5 veces más importantes que los subjetivos. Por lo que $\mathrm{K}=2.5(1-\mathrm{K})$, o sea $\mathrm{K}=0.625$.

$(1-\mathrm{K})=(1-0.625)=0.375$

Como vía de explicación del proceso aplicando la ecuación (3), el cálculo para el caso de (SJN), es:

MPLSJN=0.625(0.25694) $+0.375(0.51714)=0.354515$

El resumen de los cálculos para los diferentes niveles de preferencia se muestran en la tabla \# 9 como:

Tabla 9

Niveles de Preferencia

\begin{tabular}{lc}
\hline$\left(\mathrm{MPL}_{\mathrm{I}}\right)$ & Nivel De Preferencia \\
SJN & 0.354515 \\
$\mathrm{M}$ & 0.17548875 \\
$\mathrm{U}$ & 0.2458925 \\
$\mathrm{~A}$ & 0.22415125 \\
\hline
\end{tabular}

Fuente: elaboración propia.

Para laEtapa (4), se tiene que el Max $\{$ MPLI $\}=\operatorname{Max}\{0.354515,0.17548875,0.2458925$, $0.22415125\}=0.354515$, obteniendo como localización óptima recomendada usando el análisis en teoría clásica la localidad de (SJN).

\section{Evaluación en la incertidumbre (Lógica Difusa)}

En el entorno económico, social y tecnológico de la empresa, resulta su actividad mucho menos previsible y se encuentra en una situación más inestable que en el pasado. Esto ha motivado que desde el punto de vista macro y microeconómico, se busquen nuevas metodologías por que los sistemas económicos y las empresas están atravesando. Hoy día en la empresa se plantean problemas como la localización que exigen una toma de decisiones en un ambiente en el que los objetivos que se pretenden alcanzar aparecen de forma imprecisa. Por lo que en un nuevo contexto decisional de los análisis en la empresa, se ha pasado del determinismo a la aleatoriedad y de ahí al tratamiento de la incertidumbre (uso de lógica difusa), cuando la imprecisión se formaliza a través de situaciones en las que existe una graduación entre la pertenencia y la no pertenencia. 
En este apartado se hace una extensión a la metodología original descrita en el apartado (2), la misma se escribe en un ambiente incierto usando Lógica Difusa, de igual forma se incorpora un panel de expertos para la obtención y validación de la información usada en el análisis.

En ambiente difuso existen un número abundante de trabajos relevantes que hacen aplicaciones usando enfoques que usan métodos basados en optimización multicriterio como el de Kavita D., Shiv Prasad Y (2013) en el análisis consideran que el conocimiento base de los tomadores de decisiones es vago e impreciso hacen uso de un método intucionista usando NBT, así mismo el tratamiento de la selección de localidades de interés haciendo uso de enfoques cualitativos, además el establecimiento de orden de preferencias por similitud es abordado por Chu TC (2002a,b), de igual manera Hwang CL, Yoon K (1981) presentan una metodología basada en la evaluación de los criterios lingüísticos y asignación de pesos e importancia usando números difusos. En Young D (2006), se propone el método TOPSIS, haciendo la transformación de números difusos a nítidos, Chen CT (2001), presenta una propuesta basada en la evaluación de alternativas haciendo uso de NBT, Chou et al (2008), presentan el módulo difuso MCDM para la selección de la ubicación de un hotel turístico, Zajim Aljicevic, et al (2016), Zajim A. et al. (2018), hacen uso de MATLAB en un ambiente difuso usando NBT para proyectos de fuentes de energía renovables.

Para el tratamiento del problema objeto de esta investigación, se toman de referencia las ideas establecidas en los trabajos anteriores en los que se hace uso de optimización multicriterio, se tomaran como base los trabajos que aportan para desarrollar la presentación en un ambiente difuso la metodología de (By G), fundamentalmente los de Zadeh L.A. (1965), Kaufmann A, Gil Aluja J. (1994), González Santoyo F., Flores Romero B., Gil Lafuente A.M. (2010), González Santoyo F., Flores Romero B., Gil Lafuente A.M. (2011), Jeong J.S., Ramírez G.A. (2018), en ellos se hace uso de números borrosos-difusos triangulares (NBT), mismos que son usados para hacer la extensión de $(\mathrm{ByG})$ en ambiente difuso, para ello se establece que:

Un número difuso es representado por $\tilde{A}$, se encuentra formado por una secuencia finita o infinita de intervalos de confianza con las propiedades:

Se afecta a cada intervalo de confianza en un valor de $\alpha \in[0,1]$, de tal forma que dos intervalos de confianza diferentes no pueden tener el mismo $(\alpha)$, a este valor se le llama nivel de presunción.

Se designa por $A_{\alpha}=[r(\alpha), m(\alpha)$,$] \quad el intervalo de confianza de nivel (\alpha)$ y debe cumplir: $\alpha^{\prime}<\alpha=>A_{\alpha} \in A_{\alpha}^{\prime} \in[0,1]$

Por ello un número borroso (difuso) triangular (NBT) es una generalización del concepto de intervalo de confianza. En lugar de un único intervalo de confianza, se considera una familia que satisface las condiciones anteriores. Para este tipo de números sus funciones de pertenencia son lineales como se muestra en la figura \#1: 


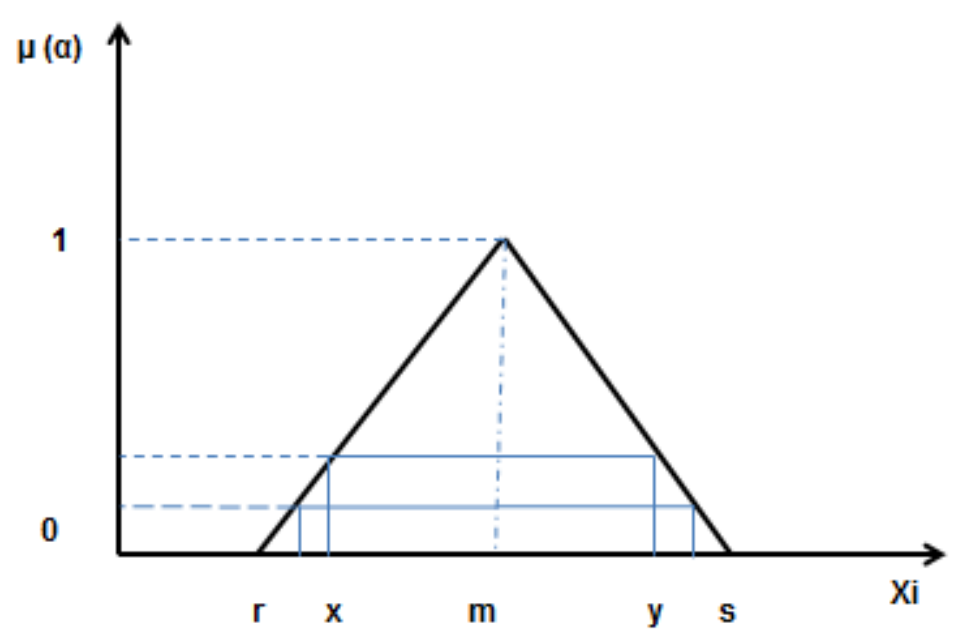

Figura 1. Representación de un NBT

Fuente: elaboración propia

Como se observa, en su representación existen tres puntos singulares que lo representan como $\tilde{A}=N B T=(r, m, s)$, con $(\mathrm{r}, \mathrm{m}, \mathrm{s}) \in \mathrm{R}, \mathrm{y}, \mathrm{r} \leq \mathrm{m} \leq \mathrm{s}$, por tanto se establece que:

Para toda $\mathrm{x} \leq \mathrm{r} ; \mu_{\alpha}(x)=0 \quad$; Para toda $\mathrm{x} \geq \mathrm{s} ; \mu_{\alpha}(x)=0 ; \mu_{A}(m)=1$

Por ello la conceptualización del uso de NBT permite estimaciones adecuadas en el campo de la economía y empresa como lo es el caso objeto de este trabajo, ya que es posible determinar (r) y (s) que corresponden a las estimaciones mínima y máxima respectivamente, y un valor $(m)$ que representa el valor de mayor presunción o el que tiene la mayor posibilidad de ocurrencia, además de la gran adaptabilidad de un NBT a la estructura del pensamiento humano. En esta investigación la información usada es representada haciendo uso de NBT.

De acuerdo con la metodología usada en este trabajo para la etapa (1), el nivel de costos es manejado en por unidad-pesos (p.u.-\$), de acuerdo con el panel de expertos, la cuantificación de los mismos es representada como se muestra en la Tabla \# 9: 
Tabla 9

Nivel de Costos difusos Asociados

\begin{tabular}{|c|c|c|c|c|}
\hline CONCIPICY & 11 & A & $M$ & SJN \\
\hline \multicolumn{5}{|l|}{ Coato (p.11 \$) } \\
\hline & $(\mathrm{r}=1,3)$ & $(x, 10, s)$ & $(\mathrm{r}+1, \mathrm{~s})$ & $(r, m, s)$ \\
\hline Manterias primas & $(7,8,5,9)$ & $(9.95,16)$ & $(9,101,10.5)$ & $(8,8.5,5)$ \\
\hline Mano ile ohra & $(9,9.4,10)$ & $(9.10,11.5)$ & $(k, g, 5,9)$ & $(8,9,9.2)$ \\
\hline 'Iranaporte & $(8,8,6,9)$ & $(9.9,6,16)$ & $(9.5,10.10 .5)$ & $(7.9 .5 .8 .4)$ \\
\hline linergin l.:éc & $(9,9.4,10)$ & $(9,9.4 .98$ & $(9,9.5 .10)$ & $(9,9,4,98)$ \\
\hline \multicolumn{5}{|l|}{ Irixa } \\
\hline Custo di kessaw & $0.10,11 \mathrm{j}$ & $(8,8.5,9)$ & $(0.5,8,8.5)$ & $(8.5,9,92)$ \\
\hline Abaskriumicalo & $(6,7,8)$ & $(6,7,8)$ & $(7,75,8)$ & $(6.5,7,75)$ \\
\hline \multicolumn{5}{|l|}{ de 11,0} \\
\hline Insumos & (8.8.5. & $(8,9,10)$ & $(6.5,7.7 .5)$ & $(8.8 .5,9)$ \\
\hline TOTAL O & $(56,61, A, 60)$ & $(58,63,68,3)$ & $(56.5,60.5,61)$ & $(55959.1,6.1)$ \\
\hline Reciprnon (1) & $\begin{array}{c}(0.1515,0.162 \mathrm{~K}, \\
\text { 0.178s })\end{array}$ & ((10146K1,0.015873,0.01724) & $(0.01525,10116528,5.0176591)$ & (110161 LO1168. (1017K8Y \\
\hline
\end{tabular}

Fuente: elaboración propia

Para el cálculo de los factores de calificación objetiva, la transformación difusa queda como se expresa en la ecuación:

$$
\widetilde{F O_{l}}=\left[\widetilde{C}_{\imath} \sum_{i=1}^{n}\left(\frac{1}{\widetilde{C}_{l}}\right)\right]^{-1}
$$

Dónde:

$\widetilde{C_{l}}=(\mathrm{r}, \mathrm{m}, \mathrm{s})=$ valor del nivel del NBT para cada localidad.

$\left(\frac{1}{\widetilde{\tilde{c}}_{l}}\right)=$ Inverso de cada nivel del NBT para cada localidad.

El cálculo del Factor de Calificación Objetiva para cada localidad de interés es realizado haciendo uso de la ecuación (5) los resultados son expresados en la tabla \# 10 como se muestra:

Tabla 10

Factor difuso de Calificación Objetivo

\begin{tabular}{cc}
\hline Localidad & $\widetilde{F O}_{\iota}$ \\
U & $(0.21435,0.24859,0.290168)$ \\
A & $(0.20715,0.24229,0.2825)$ \\
M & $(0.22107,0.25229 .0 .28771)$ \\
SJN & $(0.22779,0.25690,0.29080)$ \\
\hline
\end{tabular}

Fuente: elaboración propia. 
En la etapa (2). Para la determinación del índice de importancia relativa $\begin{array}{lll}\widetilde{\boldsymbol{W}_{\boldsymbol{J}}} & \text { y el }\end{array}$ indice de ordenamiento jerárquico $\widetilde{\boldsymbol{R}_{\boldsymbol{l}}}$ se tomó en consideración la opinión de un panel de 5 expertos. Por tratarse de opinión de seres humanos por naturaleza la información es imprecisa-aproximada, y no de cálculo realizado a través de ningún criterio matemático clásico. De acuerdo con la información proporcionada por dicho panel de expertos el cálculo para los dos índices de referencia se hizo a través del valor agregado medio difuso, la información es presentada como se muestra a continuación:

Tabla 11

Índice de Importancia Relativa en la Incertidumbre

\begin{tabular}{ll}
\hline & $\widetilde{W_{J}}$ \\
Factor (i) & 0.15 \\
Educación & 0.3 \\
Clima & 0.15 \\
Vivienda & 0.4 \\
Aceptación de la comunidad & 1 \\
Total & 1 \\
\hline
\end{tabular}

Fuente: elaboración propia

Para:

Tabla 12

Para Educación en ambiente de Incertidumbre queda:

\begin{tabular}{lc}
\hline Factor (i) & $\widetilde{\boldsymbol{R}_{\boldsymbol{\iota}}}$ \\
A & 0.4 \\
U & 0.1 \\
M & 0.1 \\
SJN & 0.4 \\
Total & 1 \\
\hline
\end{tabular}

Fuente: elaboración propia 
Tabla 13

Para para el Clima en ambiente de Incertidumbre queda:

\begin{tabular}{ll}
\hline Factor (i) & $\widetilde{\boldsymbol{R}_{\boldsymbol{l}}}$ \\
A & 0.3 \\
U & 0.4 \\
M & 0.2 \\
SJN & 0.1 \\
Total & 1 \\
\hline
\end{tabular}

Fuente: elaboración propia

Tabla 14

Para Vivienda en ambiente de Incertidumbre queda:

\begin{tabular}{ll}
\hline Factor (i) & $\widetilde{\boldsymbol{R}_{\boldsymbol{t}}}$ \\
$\mathrm{A}$ & 0.3 \\
$\mathrm{U}$ & 0.2 \\
M & 0.1 \\
SJN & 0.4 \\
Total & 1 \\
\hline
\end{tabular}

Fuente: elaboración propia.

Tabla 15

Para Aceptación de la Comunidad en ambiente de Incertidumbre:

\begin{tabular}{ll}
\hline Factor (i) & $\widetilde{\boldsymbol{R}_{\boldsymbol{\jmath}}}$ \\
A & 0.3 \\
U & 0.1 \\
M & 0.1 \\
SJN & 0.5 \\
Total & 1 \\
\hline
\end{tabular}

Fuente: elaboración propia 
De lo anterior tomando la metodología descrita en el apartado $(2),\left(\widetilde{R_{l}} ; \widetilde{W_{l}}\right)$; en un ambiente de incertidumbre es expresada como se muestra a continuación:

Tabla 16

Factores $\left(\widetilde{R_{\iota \jmath}} ; \widetilde{W_{\jmath}}\right)$

\begin{tabular}{llllll}
\hline \multicolumn{1}{c}{ FACTOR $(\mathrm{i})$} & & & $\widetilde{\boldsymbol{R}_{\boldsymbol{l}}}$ & & \multicolumn{1}{c}{$\widetilde{W_{J}}$} \\
& $\mathrm{~A}$ & $\mathrm{U}$ & $\mathrm{M}$ & $\mathrm{SJN}$ & \\
Educación & $(0.3,0.4,0.5)$ & $(0.5,0.1,0.5)$ & $(0.05,0.1,0.015)$ & $(0.3,0.4,0.42)$ & $(0.14,0.15,0.16)$ \\
Clima & $(0.2,0.3,0.4)$ & $(0.38,0.4,0.42)$ & $(0.1,0.2,0.25)$ & $0.02,0.1,0.15)$ & $(0.2,0.3,0.35)$ \\
Vivienda & $(0.1,0.3,0.4)$ & $(0.1,0.2,0.25)$ & $(0.05,0.1,0.15)$ & $(0.3,0.4,0.45)$ & $(0.14,0.15,0.16)$ \\
Aceptación de la Comunidad & $(0.2,0.3,0.4)$ & $(0.05,0.1,0.15)$ & $(0.04,0.5,0.6)$ & $(0.4,0.5,0.6)$ & $(0.3,0.4,0.5)$ \\
\hline
\end{tabular}

Fuente: elaboración propia

De igual forma que el análisis anterior, los Factores Subjetivos son calculados con la ecuación (6) haciendo la transformación para el uso de Lógica Difusa, los resultados para cada localidad quedan como se muestra:

$$
\widetilde{F S}_{\iota}=\sum_{i=1}^{n} \widetilde{R_{\imath \jmath}} \widetilde{W_{\jmath}}
$$

Tabla 17

Factores Subjetivos $(\widetilde{F S})$.

\begin{tabular}{lc}
\hline$\left(\widetilde{F S}_{l}\right)$. & Nivel de Importancia \\
$\mathrm{A}$ & $(0.234,0.315,0.46)$ \\
$\mathrm{U}$ & $(0.141,0.205,0.286)$ \\
$\mathrm{M}$ & $(0.046,0.13,0.1435)$ \\
SJN & $(0.208,0.35,0.4917)$ \\
\hline
\end{tabular}

Fuente: elaboración propia

Para las etapas (3 y 4). Haciendo uso del mismo criterio que para el caso clásico, para la determinación de $\widetilde{M P L}_{\imath}$ difusa, se hace uso de la información expresada en un ambiente difuso obtenida a través de las ecuaciones 5 y 6 , por lo que el criterio para determinar la decisión más adecuada para localizar la planta es obtenido a través del uso de la ecuación (7) mostrada como: 
$\operatorname{maxM} P L_{I}=k\left(\widetilde{F O_{I}}\right)+(1-k)\left(\widetilde{F S_{l}}\right)$

Si. $\mathrm{K}=0.625 ;(1-\mathrm{K})=(1-0.625)=0.375$.

Por tanto, los niveles de preferencia obtenidos usando (6), son:

Tabla 18

Niveles de Preferencia Fuzzy

\begin{tabular}{ll}
$\widetilde{M P L}_{I .}$ & \multicolumn{1}{c}{ Nivel de Preferencia } \\
$\mathrm{U}$ & $(0.066271,0.092411,0.125385)$ \\
$\mathrm{A}$ & $(0.100696,0.133268,0.190015)$ \\
$\mathrm{SJN}$ & $(0.092236,0.147306,0.20255)$ \\
$\mathrm{M}$ & $(0.031066,0.064518,0.071791)$ \\
\hline
\end{tabular}

Fuente: elaboración propia

Para la etapa (4), la selección de la mejor localización es asociada con la localidad de SJN como se muestra a continuación:

$\operatorname{Max}\left\{\widetilde{M P L}_{I}\right\}=\{(0.066271,0.092411,0.125385),(0.100696,0.133268,0.190015),(0.092236$, $0.147306,0.20255),(0.031066,0.064518,0.071791)\}=(0.092236,0.147306,0.20255)$. 


\section{Conclusiones}

Del análisis realizado, para la determinación de la localización de una empresa haciendo uso de la metodología de (ByG) con información determinística y en la incertidumbre (Lógica Difusa), descrita en los apartados anteriores, se concluye que la incorporación y determinación de factores localizacionales objetivos y subjetivos, así como que la incorporación de la apreciación de un panel de 5 expertos en el análisis de los factores básicos localizacionales de referencia lleva a una toma de decisiones más eficiente y eficaz en la selección del mejor sitio (localidad) para instalar una empresa en la localidad que de pleno cumplimiento del conjunto de $\{\mathrm{FBLi}, \mathrm{i}=1,2, \ldots, \mathrm{n}\}$, para ello la metodología propuesta para el análisis en la certeza como en la incertidumbre (Lógica Difusa) cumple plenamente para recomendar la población de San Juan Nuevo Parangaricutiro en el Estado de Michoacán, México como la localidad óptima que garantiza el mínimo costo operativo y el mayor nivel de beneficio al inversionista.

En el estudio se da una mayor importancia a la disponibilidad, costo, cercanía y suficiencia de las materias primas e insumos en el horizonte de planeación de la empresa de interés, así mismo se concluye que el uso de la Lógica Difusa es una herramienta que garantiza una toma de decisiones alternativa a las usadas en la teoría clásica escrita en una lógica bivalente, en la cual el espacio se soluciones es expresado con un valor puntual en la recta de los números reales, mientras que la evaluación en la incertidumbre se hace uso de la lógica difusa, la respuesta siempre será un intervalo en el que aparecerán infinitas soluciones dependiendo de las particiones que se realicen en el intervalo de solución representado por un número difuso para el caso es NBT por lo que en el intervalo solución solamente se presentan 3 valores expresando el límite inferior, el valor más posible y el límite superior en el que se da la solución del problema, esto en virtud de que se trabaja con una base de conocimiento escrita en una lógica multivalente, a diferencia del caso clásico, por ello este tipo de análisis resulta más eficiente y eficaz.

Esto implica que al tener la empresa una localización óptima contribuye a que sea más competitiva en el mercado local, regional, nacional e internacional en el área económica que tiene relación.

De lo anterior se proponen como futuras líneas de investigación en este campo, hacer extensiones a métodos de optimización existentes en el campo de la Investigación de Operaciones haciendo propuestas de transformación al ambiente difuso, así como comparación de eficiencia y eficacia entre los resultados obtenidos en el ambiente clásico y difuso. 


\section{Referencias}

A.Klose. A. Drexl. (2005). Facility location models for distribution system design. European Journal of Operational Research. 162 (1), 4-29. https://doi.org/10.1016/j.ejor.2003.10.031

Antony B., Goyal V., Gupta A., Nagarajan V., (2010). A Plant Location Guide for the Unsure: Aproximation Algorithms for Min-Max Location Problems. Mathematics of Operations Research. 35(1), 79-101. https://doi. org/10.1287/moor.1090.0428

Bojic S. et al. (2018). Location problem of lignocellulosic bioethanol plant. Case study Serbia. Journal of Cleaner Production. 172, 971 - 979. https://doi.org/10.1016/j.jclepro.2017.10.265

Brown, P.A, Gibson D.F. (1972). A quantified model for facility site selection, Application to a multiplant location problema. AIIE Transactions 4(1), 1-10. https://doi.org/10.1080/05695557208974822

Castro García F, González Santoyo F, Pacheco D.C. (2010). Lógica difusa aplicada como una herramienta adicional a los medios convencionales de localización. Caso localización de un negocio de servicios. XVI SIGEF Congress. Morelia. México.

Chen CT (2001). A fuzzy approach to select the location of the distribution center. Fuzzy Set Syst 118(1), 65-73. https://doi.org/10.1016/s0165-0114(98)00459-X

Chou SY, Chang YH, Shen CY (2008). A fuzzy simple additive weighting system under group decision-making for facility location selection with objective /subjective attributes. European Journal of Operational Research, 189(1),132-145. https://doi.org/10.1016/j.ejor.2007.05.006

Chu TC (2002) Facility location selection using fuzzy TOPSIS under group decisions. International Journal of Uncertain Fuzziness Know-Based System, 10(6),687-701. https://doi.org/10.1142/s0218488502001739

Chu TC (2002). Selecting plant location via a fuzzy TOPSIS approach. The International Journal of Advanced Manufacturing Technology, 20(11), 859-864. https://doi.org/10.1007/s001700200227

Dilworth, J.B. (1993). Production and operations Management. Mc. Graw Hill. US.

Fazel Zarandi M., Beck J.C. (2012). Using logic -based Bemders Descomposition to solve the Capacity - and Distance-Constrained Plant Location Problem. INFORMS Journal on Computing, 24(3), 387-398. https://doi. org/10.1287/ijoc.1110.0458

Flores Garrido L., Oliva San Martín C. (2016). Algorithms for the problem of location of plants and distribution centers maximizing beneficit. Ingeniare, Revista chilena de ingeniería 24(3),508-516.

Garret y Silver (1973). Production Management Analysis. Harcourt Brace Jovanovich International Edition.

González Santoyo, F. (1985). Los proyectos en la industrialización forestal. Editorial Universitaria de la Universidad Michoacana de San Nicolás de Hidalgo. Morelia México.

González Santoyo F, Flores Romero B., Gil Aluja A.M. (2010). Modelos y Teorías para la Evaluación de Inversiones Empresariales. Fegosa-Ingeniería Administrativa S.A. de C.V., IAIDRES, UMSNH. México.

González Santoyo F, Flores Romero B., Gil Aluja A.M. (2011). Procesos para la Toma de Decisiones en un entorno Globalizado. Editorial Universitaria Ramón Areces. España.

Hwang CL, Yoon K (1981). Multiple attribute decisión making methods and applications: a state of the art survey. Springer, Berlin.

Jeong J.S., Ramírez G.A. (2018). Optimizing the location of biomass plant with a fuzzy-decision-making trial and evaluation laboratory (F-DEMATEL) and multi-criteria spatial decision assessment and long-term sustainability. Journal of Cleaner Production. 182, 509-520. https://doi.org/10.1016/j.jclepro.2017.12.072

Kaufmann A., Gil Aluja J, Terceño Gómez A. (1994). Matemática para la Economía y la Gestión de Empresas. Vol I. Aritmética de la Incertidumbre. Ediciones Foro Científico. Barcelona.

Kavita D., Shiv Prasad Y (2013). A multicriteria intuitionalistic fuzzy group decisión making for plant location selection with ELECTRE method. The International Journal of Advanced Manufacturing Technology, 66, 9-12. https://doi.org/10.1007/s00170-012-4400-0 
Klir, G.J., Bo Yuan (1995). Fuzzy sets and Fuzzy Logic (Theory and Aplications).Upper Saddle River, Nueva Yersey. Prentice Hall Inc.

Machuca, J.A.D., et al. (1994). Aspectos estratégicos en la producción y los servicios. Mc Graw Hill. Méx.

Medina J.R., Romero R.L. Pérez G.A. (2009). Critical review and adequation criteria used in this decisión. Revista Mexicana de Ingeniería Química 18(3), 271-274.

Melkote, S. \& M. Daskin (2001). Capacitated facility/network desing problems. European Journal of Operational Research. 129 (3), 481-495. https://doi.org/10.1016/s0377-2217(99)00464-6

ONU, (1958). Manual de proyectos de desarrollo económico. ONU México D.F.

Tawfik, L., Chauvenel, A.M. (1993). Administración de la Producción. Mc Graw Hill. México.

Zajim Aljicevic, Aleksandra Kestic, Nedis Dautbasic, Gunary Karly (2016). Model of Fuzzy Logic for Selection Infrastructural Investment Project of Wind farm Locations. 27 Th DAAAM International Symposium on Inte1ligent Manufacturing and Automation. Vienna Austria. https://doi.org/10.2507/27th.daaam.proceedings.107

Zajim Aljicevic, Aleksandra Kestic, Nedis Dautbasic (2018). Selecting location for infrastructural investment project in renewable sources of energy using MATLAB and fuzzy logic.Tome XVI, Fascicule 2. Unniversity Politehnica Timisoara. Romania.

Young D (2006). Plant location selection base on fuzzy TOPSIS. The International Journal of Advanced Manufacturing Technology 28(7), 839-844. https://doi.org/10.1007/s00170-004-2436-5

Zadeh L.A. (1965). Fuzzy Sets. Information and Control. 8(3). 338-353. 